\title{
ESTUDO CINÉTICO DA REMOÇÃO DE DICLOFENACO DE SÓDIO EM SOLUÇÃO AQUOSA POR ADSORÇÃO EM ARGILA ORGANOFÍLICA SPECTROGEL
}

\author{
P. K. BORGES ${ }^{1}$, G. S. MAIA ${ }^{2}$, M. G. C. DA $\operatorname{SILVA}^{3}$ e M. G. A. VIEIRA ${ }^{3}$ \\ ${ }^{1}$ Bolsista de Iniciação Científica - CNPq \\ ${ }^{2}$ Mestranda - LEA/DDPP/FEQ/UNICAMP \\ ${ }^{3}$ Docente - DDPP/FEQ/UNICAMP
}

Departamento de Desenvolvimento de Processos e Produtos - Faculdade de Engenharia Química - UNICAMP, Av. Albert Einstein, 500 - Campinas, SP - CEP: 13083-852

E-mail para contato: melissagav@feq.unicamp.br

\begin{abstract}
RESUMO - Este trabalho teve como objetivo analisar a cinética de adsorção do fármaco diclofenaco de sódio presente em soluções aquosas utilizando a argila organofílica comercial Spectrogel tipo C. Os modelos matemáticos de pseudoprimeira ordem, pseudossegunda ordem e difusão intrapartícula foram utilizados no ajuste aos resultados experimentais. O modelo de pseudossegunda ordem apresentou melhor ajuste, com elevado $\mathrm{R}^{2}$ e baixo Desvio Médio Relativo. O modelo de difusão intrapartícula indicou que esta etapa participa do mecanismo de adsorção, podendo interferir na velocidade do processo. Sendo a maior capacidade de adsorção observada no ensaio cinético de maior concentração.
\end{abstract}

\section{INTRODUÇÃO}

Nos últimos anos, a produção de materiais farmacêuticos tem aumentado consideravelmente e a sua detecção no meio ambiente foi registrada por diversos pesquisadores, seja em efluentes domésticos ou industriais bem como em águas naturais (Jones, 2007). Esses compostos xenobióticos são mineralizados nas Estações de Tratamento de Efluentes (ETEs), porém, a eliminação total desses microcontaminantes por métodos convencionais é incompleta (Radjenovic et al., 2007). Sendo a detecção em efluentes (Andreozzi et al., 2003; Radjenovic et al., 2007; Ternes, 1998), biossólidos (Radjenović et al., 2009) e águas de superfície (Jux et al., 2002; Fernández et al., 2010) comum. O diclofenaco de sódio é um medicamento amplamente utilizado devido suas propriedades analgésicas e antirreumáticas e quando presente no meio ambiente pode resultar em potenciais efeitos tóxicos às algas e peixes (Al-Rajab et al., 2010).

A adsorção é um método alternativo de tratamento, eficiente na eliminação de substâncias não desejáveis e quando combinada com um material adsorvente de baixo custo, abundante e eficaz, tal método se torna ainda mais atrativo. A argila é um material natural de baixo custo com eficiência comprovada na remoção de contaminantes. O objetivo deste experimento é estudar a cinética de adsorção de diclofenaco de sódio em argila organofílica Spectrogel tipo $\mathrm{C}$ em banho finito. 


\section{MATERIAIS E MÉTODOS}

\subsection{Material adsorvente}

O material adsorvente utilizado na pesquisa foi a argila organofílica Spectrogel tipo C doada pela empresa Spectrochem ${ }^{\circledR}$.

\subsection{Adsorbato}

O diclofenaco de sódio foi obtido comercialmente através da empresa GEOLAB ${ }^{\circledR}$, com teor de pureza de 99,58\%. As soluções foram feitas com água deionizada.

\subsection{Metodologia}

Em testes preliminares, foram avaliadas as melhores condições para se realizar os ensaios de cinética. Assim, nas condições propostas, foram realizados 3 ensaios cinéticos nas concentrações de $0,03,0,20$ e $0,37 \mathrm{mmol} . \mathrm{L}^{-1}$. Os ensaios foram realizados em frascos Erlenmeyers onde $50 \mathrm{~mL}$ de solução de diclofenaco de sódio foram colocadas em contato com $0,75 \mathrm{~g}$ de argila organofílica, em temperatura constante de $25 \pm 2{ }^{\circ} \mathrm{C}$ durante um período de $24 \mathrm{~h}$ sob agitação constante. Alíquotas da solução foram retiradas em intervalos de tempos pré-determinados, centrifugadas e diluídas para a leitura no espectrofotômetro UV-VIS no comprimento de onda de $276 \mathrm{~nm}$.

A capacidade de adsorção do fármaco foi calculada conforme a Equação 1:

$$
q=\frac{\left(c_{0}-c\right) v}{m}
$$

Em que: q é a capacidade de adsorção do fármaco (mmol.g $\left.\mathrm{g}^{-1}\right) ; \mathrm{c}_{0}$ é a concentração inicial do fármaco $\left(\mathrm{mmol} . \mathrm{L}^{-1}\right)$; c é a concentração final do fármaco $\left(\mathrm{mmol} . \mathrm{L}^{-1}\right)$; v é o volume (L); m é a massa do adsorvente (g).

Os modelos de pseudoprimeira e pseudossegunda ordem e o modelo de difusão intrapartícula foram utilizados no ajuste aos resultados. O primeiro modelo é aplicado quando há uma resistência à transferência de massa pela camada externa do material adsorvente. Se for considerado que no instante $\mathrm{t}=0$ s não há adsorbato em contato com o sólido, a Equação 2 descreve o modelo:

$$
q=q_{e}\left(1-e^{-k_{1} t}\right)
$$

Sendo $\mathrm{q}_{\mathrm{e}}$ a quantidade de soluto adsorvido por unidade de massa de adsovente no equilíbrio (mmol.g $\left.\mathrm{g}^{-1}\right), \mathrm{k}_{1}$ é constante da taxa de adsorção de pseudoprimeira ordem $\left(\mathrm{min}^{-1}\right)$ e $\mathrm{t}$ é o tempo (min). 
Se considerar as mesmas condições iniciais para o modelo pseudossegunda ordem, a Equação 3 descreve o modelo:

$$
\frac{q}{q_{e}}=\frac{k_{2} \cdot q_{e} \cdot t}{\left(1+k_{2} \cdot q_{e} \cdot t\right)}
$$

Sendo $\mathrm{k}_{2}$ a constante cinética do modelo de pseudossegunda ordem (g/mmol.min). Esse modelo sugere a etapa limitante do processo de adsorção depende de reações físico-químicas entre o fármaco e o material adsorvente.

O modelo de difusão intrapartícula assume que o processo de adsorção é dominado pela difusão interna através do transporte no poro ou na superfície, e este processo é a parte lenta. A Equação 4 representa o modelo:

$$
q=k_{i} \cdot(t)^{0,5}+c
$$

Em que $\mathrm{k}_{\mathrm{i}}$ é a constante de difusão intrapartícula ( $\mathrm{mmol} / \mathrm{g} \cdot \mathrm{min}^{0,5}$ ) e c é uma constante que representa a espessura da camada limite $(\mathrm{mmol} / \mathrm{g})$

\section{RESULTADOS E DISCUSSÃO}

Os modelos matemáticos de pseudoprimeira, pseudossegunda ordem e difusão intrapartícula foram utilizados nos ajustes aos resultados experimentais e as curvas estão apresentadas nas Figuras 1, 2 e 3. Os modelos são aplicados para descrever a relação adsorbato/adsorvente.

Figura 1 - Ajuste das curvas cinéticas para as soluções de 0,37 mmol. $\mathrm{L}^{-1}$ aos modelos de (A) pseudoprimeira e pseudossegunda ordem e (B) difusão intrapartícula.

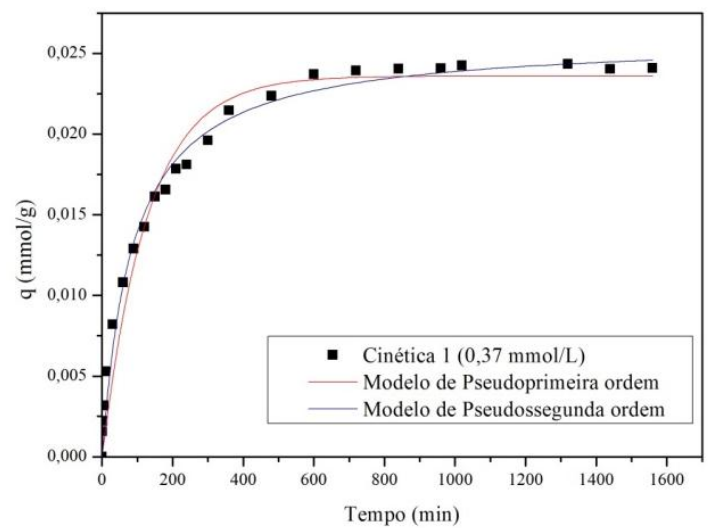

(A)

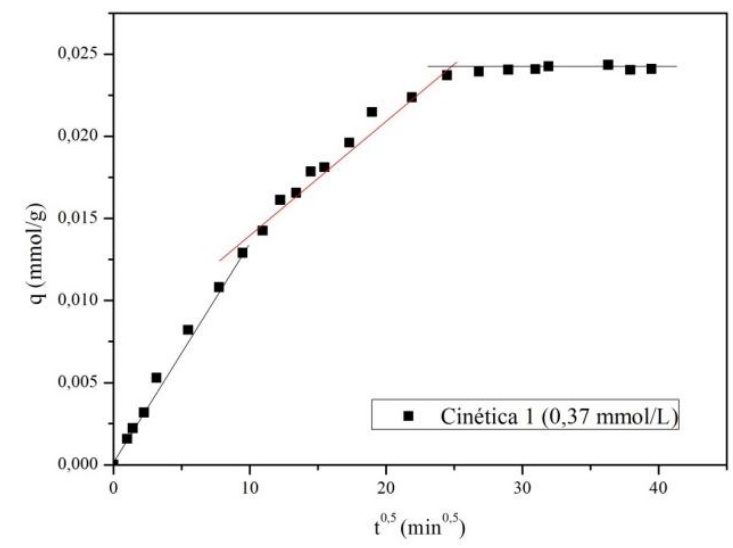

(B) 
Figura 2 - Ajuste das curvas cinéticas para as soluções de 0,20 mmol. $\mathrm{L}^{-1}$ aos modelos de (A) pseudoprimeira e pseudossegunda ordem e (B) difusão intrapartícula.

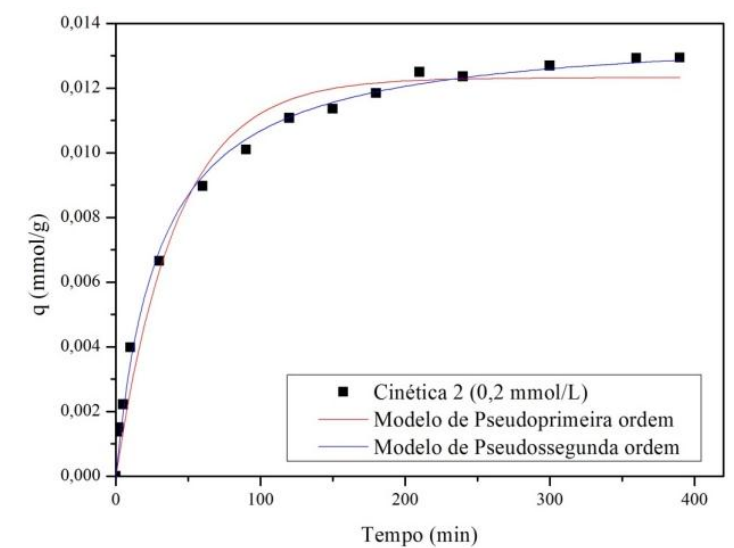

(C)

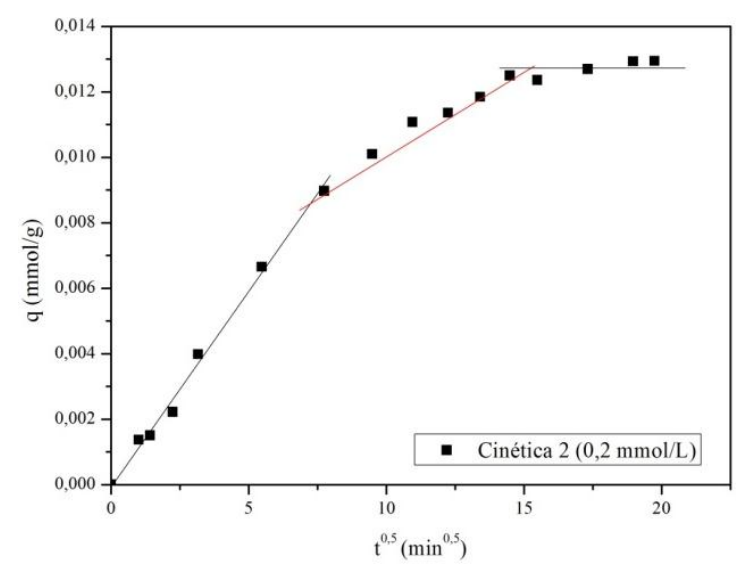

(D)

Figura 3 - - Ajuste das curvas cinéticas para as soluções de $0,03 \mathrm{mmol} . \mathrm{L}^{-1}$ aos modelos de (A) pseudoprimeira e pseudossegunda ordem e (B) difusão intrapartícula.

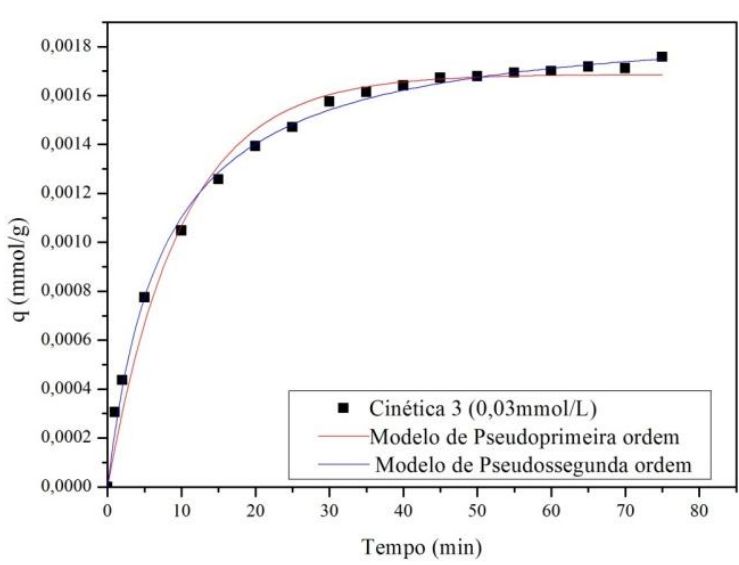

(E)

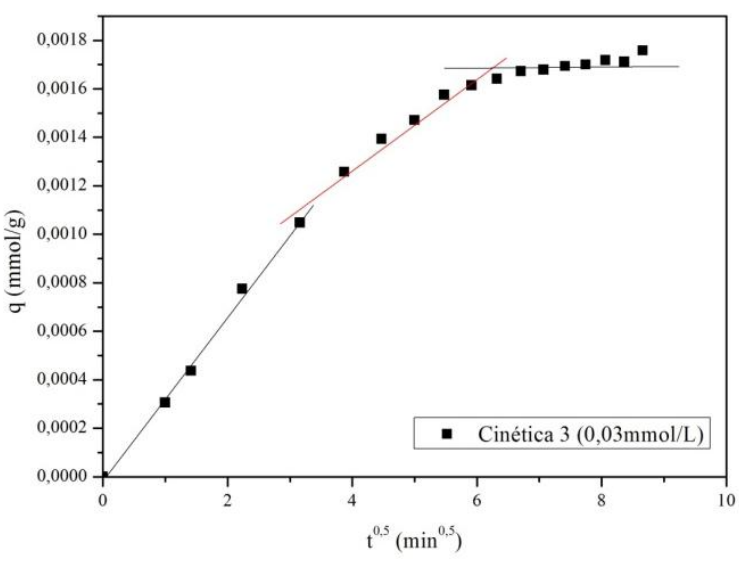

$(\mathrm{F})$

Ao analisar as curvas obtidas pode-se observar que quanto maior a concentração de adsorbato, maior foi a capacidade adsortiva obtida. Para a concentração de $0,37 \mathrm{mmol} / \mathrm{L}$, a capacidade adsortiva foi de $0,0225 \mathrm{mmol} . \mathrm{g}^{-1}$, enquanto para a concentração de $0,20 \mathrm{mmol} . \mathrm{L}^{-1}$, a capacidade de remoção foi em torno de $0,013 \mathrm{mmol} \cdot \mathrm{g}^{-1}$ e para a menor concentração 0,03 mmol. $\mathrm{L}^{-1} \mathrm{o}$ valor adsorvido foi de $0,0017 \mathrm{mmol} . \mathrm{g}^{-1}$.

Os coeficientes de determinação $\left(\mathrm{R}^{2}\right)$ e o desvio médio relativo (DMR) foram obtidos para todas as curvas, estes parâmetros podem ser utilizados para estabelecer qual modelo descreve melhor os resultados experimentais. Os parâmetros obtidos estão apresentados na Tabela 1. Comparando os modelos de pseudoprimeira e pseudossegunda ordem observa-se que os $\mathrm{R}^{2}$ são satisfatórios para ambos os modelos indicando que os dois podem ser utilizados na descrição dos resultados experimentais, contudo, os valores de DMR obtidos são ligeiramente superiores para o modelo de pseudoprimeira ordem com exceção do valor 
observado na cinética 3. O maior DMR obtido no estudo da concentração 0,03 mmol. $\mathrm{l}^{-1}$ para o modelo de pseudossegunda ordem pode estar relacionado ao maior distanciamento entre o q $\mathrm{q}_{\exp }$ e o $\mathrm{q}_{\text {modelo }}$ nos pontos iniciais.

Tabela 1 - Parâmetros obtidos com os ajustes dos modelos para as três concentrações utilizadas

\begin{tabular}{|c|c|c|c|c|}
\hline \multirow{2}{*}{ Modelo } & \multirow{2}{*}{ Parâmetro } & \multicolumn{3}{|c|}{ Concentrações (mmol/L) } \\
\hline & & 0,37 & 0,20 & 0,03 \\
\hline \multirow{3}{*}{$\begin{array}{c}\text { Pseudoprimeira } \\
\text { ordem }\end{array}$} & $\mathrm{R}^{2}$ & 0,969 & 0,979 & 0,986 \\
\hline & $\operatorname{DMR}(\%)$ & 20,4 & 16,4 & 7,3 \\
\hline & $\mathrm{k}_{1}\left(\min ^{-1}\right)$ & 0,0078 & 0,0241 & 0,1006 \\
\hline \multirow{3}{*}{$\begin{array}{c}\text { Pseudossegunda } \\
\text { ordem }\end{array}$} & $\mathrm{R}^{2}$ & 0,986 & 0,994 & 0,997 \\
\hline & $\operatorname{DMR}(\%)$ & 20,3 & 14,9 & 13,8 \\
\hline & $\mathrm{k}_{2}\left(\min ^{-1}\right)$ & 0,45 & 2,447 & 70,172 \\
\hline \multirow{4}{*}{$\begin{array}{c}\text { Difusão } \\
\text { intrapartícula }\end{array}$} & $\mathrm{R}^{2}$ & 0,976 & 0,979 & 0,969 \\
\hline & DMR $(\%)$ & 2,90 & 1,27 & 1,31 \\
\hline & $\begin{array}{c}\mathrm{k}_{\mathrm{i}} \\
\left(\mathrm{mmol} / \mathrm{g} \cdot \min ^{1 / 2}\right)\end{array}$ & 0,0006 & 0,0005 & 0,0002 \\
\hline & $\mathrm{c}(\mathrm{mmol} / \mathrm{g})$ & 0,0083 & 0,0053 & 0,0007 \\
\hline
\end{tabular}

Pode-se sugerir, então, que o modelo de pseudossegunda ordem melhor descreve os resultados experimentais em todas as concentrações avaliadas, indicando que a quimissorção ocorre no processo de adsorção do diclofenaco de sódio pela argila Spectrogel tipo C. Esse resultado é esperado e condizente com os encontrados na literatura (Luna et al., 2017; Antunes et al., 2012). As constantes cinéticas $\mathrm{k}_{1}$ e $\mathrm{k}_{2}$ são maiores quanto menor for a concentração da solução, indicando que quanto menor a concentração inicial, mais rapidamente irá acontecer a adsorção.

O modelo de difusão intrapartícula, Figuras 1-B, 2-B e 3-B, apresentou valores satisfatórios de $\mathrm{R}^{2}$ e baixos DMR, para o cálculo deste utilizou-se os pontos presentes no segundo estágio (reta vermelha) linearizados. Sugerindo-se, assim, que a difusão intrapartícula pode interferir na velocidade do processo de adsorção. A constante cinética $\mathrm{k}_{\mathrm{i}}$ apresentou um valor baixo para todas as concentrações utilizadas sendo a mais baixa observada na menor concentração. A constante c indica a proximidade da camada limite de adsorção, e analisando os valores obtidos, conclui-se que quanto maior for a concentração de fármaco, maior será a camada limite.

\section{CONCLUSÃO}

Os resultados obtidos foram satisfatórios e condizentes aos encontrados na literatura com elevados $\mathrm{R}^{2}$, valores superiores a $0,95 \mathrm{em}$ todos os casos. Ambos os modelos de pseudoprimeira e pseudossegunda ordem podem ser utilizados para descrever os resultados experimentais. Contudo, devido ao menor DMR encontrado pelo modelo de pseudossegunda ordem sugere-se que este melhor descreve os resultados obtidos, indicando que a quimissorção ocorre no processo de adsorção do diclofenaco de sódio. Por fim, o modelo de difusão intrapartícula também se mostrou válido com elevado coeficiente de determinação e baixos DMR indicando que a difusão intrapartícula pode interferir na velocidade do processo. 


\section{REFERÊNCIAS BIBLIOGRÁFICAS}

AL-RAJAB, A.A.; SABOURIN, L.; LAPEN, D.; TOPP, E. The non-steroidal antiinflammatory drug diclofenac is readily biodegradable in agricultural soils. Science of The Total Environment, v. 409, p. 78-82, 2010.

ANDREOZZI, R.; RAFFAELE, M.; NICKLAS, P. Pharmaceuticals in STP effluents and their solar photodegradation in aquatic environment. Chemosphere, v. 50, p. 1319-1330, 2003.

ANTUNES, M.; ESTEVES, V. I.; GUÉGAN, R.; CRESPOA, J. S.; FERNANDES, A. N.; GIOVANELA, M. Removal of diclofenac sodium from aqueous solution by Isabel grape bagasse. Chemical Engineering Journal, v. 192, p. 114-121, 2012.

FERNÁNDEZ, C.; GONZALES-DONCEI, M.; PRO, J.; CARBONELI, G.; TARAZONA, J. $\mathrm{V}$. Occurrence of pharmaceutically active compounds in surface Waters of the henaresjaramatajo river system (Madrid, Spain) and a potential risk characterization. Science of The Total Environment, v. 408, p. 543-551, 2010.

JONES, O.A.H.; VOULVOULIS, N.; LESTER, J.N. The occurrence and removal of selected pharmaceutical compounds in a sewage treatment works utilizing activated sludge treatment. Environmental Pollution, v. 145, n. 3, p. 738-744, 2007.

JUX, R.; BAGINSKI, R.; ARNOLD, H.; KRONKE, M.; SENG, P. Detection of pharmaceutical contamination of rivers, pond, and tap water from Cologne (Germany) and surroundings. International journal of hygiene and Environment Health, v. 205, p. 393-398, 2002 .

LUNA, M. D. G.; MURNIATI; BUDIANTA, W.; RIVIERA, K. K. P.; ARAZO, R. O. Removal of sodium diclofenac from aqueous solution by adsorbents derived from cocoa pod husks. Journal of Enrivonmental Chemical Engineering, 2017.

RADJENOVIC, J.; PETROVIC, M.; BARCELÓ, D. Analysis of pharmaceuticals in wastewater and removal using a membrane bioreactor. Analytical and Bioanalytical Chemistry., v. 387, n. 4, p. 1365-1377, 2007.

RADJENOVIĆ, J.; PETROVIĆ, M.; BARCELÓ, D. Fate and distribution of pharmaceuticals in wastewater and sewage sludge of the conventional activated sludge (CAS) and advanced membrane bioreactor (MBR) treatment. Water Research, v. 43, n. 3, p. 831-841, 2009.

TERNES, T. A. Occurrence of drugs in German sewage treatment plants and rivers. Water Research., v. 32, n. 11, p. 3245-3260, 1998.

\section{AGRADECIMENTOS}

Ao CNPq, à Capes e à FAPESP (Proc. 2016/05007-1) pelo suporte financeiro e à Geolab e à Spectrochem pela doação do fármaco e da argila Spectrogel Tipo C, respectivamente. 